\section{Cytomegalovirus and the eye}

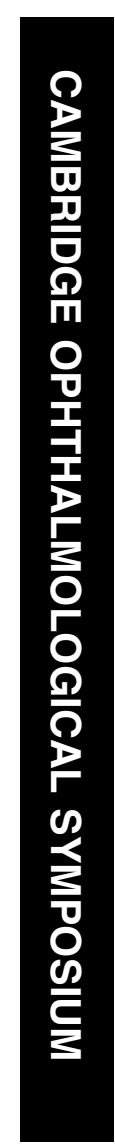

lasting 1-3 weeks associated with a transient lymphocytosis and abnormal liver function tests. During primary infection, there is a transient viraemia by which the virus reaches all parts of the body, including the bone marrow.

When CMV infects a cell, the viral DNA genome migrates to the cell nucleus where the subsequent course of the infection depends upon the state of activation/differentiation of the cell. In certain cell types that are fully permissive for viral replication such as fibroblasts or endothelial cells, there is a temporally regulated cascade of virus gene expression. The first viral genes to be transcribed are immediate early genes that encode viral DNA-binding proteins, which regulate the expression of other viral genes, followed by early genes that initiate viral genomic DNA replication, followed by late genes that encode viral structural proteins, which assemble to form new virus particles.

In contrast, in the bone marrow CMV infects $\mathrm{CD} 34+$ myeloid progenitor cells in which specific human cellular DNA-binding proteins in the nucleus bind to the CMV immediate-early promoter and inhibit transcription, thereby blocking the production of infectious particles. Thus in these non-permissive myeloid progenitor cells, the virus becomes latent - the viral genome persists in the nucleus but expresses only a very limited number of viral genes - and is therefore very difficult for the immune system to detect and eradicate. As the latently infected myeloid progenitor cells divide and differentiate into monocytes, the virus is carried out of the bone marrow in circulating monocytes and subsequently in tissue macrophages and dendritic cells throughout the body. When latently infected macrophages and dendritic cells subsequently become highly activated (eg, as part of their appropriate response to some other intercurrent bacterial or viral infection), the conditions inside the nucleus change. The human cellular DNAbinding proteins that previously bound to the
Addenbrooke's Hospital, Cambridge, UK

Correspondence: A Carmichael, Consultant Physician, Infectious Diseases Service, Addenbrooke's Hospital, Hills Road, Cambridge CB2 OQQ, UK

Tel: + 44 (0)1223 245151; Fax: + 44 (0)1223 256252 E-mail: andrew.carmichael@ addenbrookes.nhs.uk

Received: 19 October 2011 Accepted: 21 October 2011 Published online: 16 December 2011

Based on a presentation at the 2011 Cambridge Ophthalmology Symposium. 
CMV immediate-early promoter disappear, permitting the virus to reactivate from latency; now the full cascade of gene expression leads to production of infectious virus particles that are released from epithelial surfaces in the pharynx, breast, and genital tract. Thus in healthy carriers of $\mathrm{CMV}$, asymptomatic reactivation and shedding of CMV occur intermittently throughout life especially during intercurrent illnesses. Virus reactivation is normally controlled by the immune system, but in immuno-compromised patients CMV can cause serious disease.

\section{The immune response against $\mathrm{CMV}$}

In response to primary CMV infection, anti-CMV antibody is produced initially as IgM followed by IgG which persists life-long. However, the most important immune responses against CMV are CD4 $+\mathrm{T}$ cells and CD8 + T cells - patients who have severely impaired T-cell responses can develop serious CMV disease despite having anti-CMV antibody (eg, in advanced HIV infection).

T cells have on their surface a highly specific antigen receptor, which is generated by VDJ gene recombination during T-cell development in the thymus. The antigen receptor recognizes short peptides, which are presented by MHC molecules on the surface of virus-infected cells; $\mathrm{CD} 4+\mathrm{T}$ cells recognize peptides derived from extracellular proteins presented by MHC Class II molecules, whereas CD8 $+\mathrm{T}$ cells recognize peptides derived from intracellular proteins presented by MHC Class I molecules. When the T-cell receptor recognizes a specific peptide presented on a dendritic cell in a lymph node, the T-cell undergoes sustained clonal proliferation and differentiation into highly activated effector $\mathrm{T}$ cells that secrete cytokines and/or kill virus-infected cells. Once the initial virus infection has been controlled, most of the activated $\mathrm{T}$ cells die by apoptosis, but the remainder persist as an expanded population of circulating virus-specific memory $T$ cells that can undergo rapid proliferation when they encounter the virus again.

The CD8 + T-cell response against CMV typically focuses on a small number of peptides derived from relatively small number of viral proteins, such as the immediate-early protein IE-1 and the tegument phosphoprotein pp65. The precise peptide that is recognized depends upon which MHC Class I molecules a person possesses, because a given MHC Class I molecule preferentially binds 8-10 amino-acid peptides that have characteristic amino acid(s) at key positions (eg, HLA-B27 preferentially binds peptides that possess an arginine residue at position 2). During primary CMV infection, there is activation of a number of different
T-cell clones that recognize an individual viral peptide, but within a few weeks there is progressive clonal focusing with contraction of lower affinity clones due to apoptosis, but sustained expansion of a few dominant high-affinity clones that persist at high levels for many years. When independent $\mathrm{T}$-cell clones that recognize a given virus peptide are obtained from different healthy carriers of $\mathrm{CMV}$, the T-cell clones often show striking similarity in their T-cell receptor structure because this confers high affinity for the particular viral peptide. Faced with this strong sustained CD8 + T-cell response, during its evolution, CMV has acquired a set of genes that encode viral proteins, which specifically interfere with the MHC Class I antigen presentation pathway (by interfering with transport of peptides into the endoplasmic reticulum, or blocking the movement of peptide-MHC complexes to the cell surface, or ejecting peptide--MHC complexes from endoplasmic reticulum into the cytoplasm for premature degradation). During viral reactivation from latency in an antigen-presenting cell such as a dendritic cell or macrophage, these immune evasion genes may camouflage the virus for just enough time to infect adjacent cells and reach an epithelial surface and thereby transmit infection to another person, before the virus-specific CD8 $+\mathrm{T}$ cells arrive and kill the virus-infected cells

\section{Clinical settings of CMV retinitis}

CMV retinitis occurs in patients who have failed to generate a primary $\mathrm{T}$-cell response against the virus (in solid organ transplantation or allogeneic bone marrow transplantation) or in patients who are carriers of CMV but whose previously effective CMV-specific T-cell response has decreased owing to disease (AIDS) or immuno-suppressive treatment (including chemotherapy for malignant disease).

In solid organ transplantation, the risk is greatest in CMV-negative patients who receive an organ from a CMV-positive donor, because the virus is transferred into the recipient at the same time that the recipient's $\mathrm{T}$ cells are deliberately suppressed by immuno-suppressive drugs to prevent allograft rejection. Although such $\mathrm{D}+/ \mathrm{R}-$ recipients normally received prophylactic anti-CMV treatment for the first 3 months after transplantation, when this antiviral prophylaxis ceases CMV viraemia usually follows often at high levels. In this setting, CMV retinitis may occur at a median 9 months (range 4 months-13 years) after transplantation, is often bilateral, and requires prolonged antiviral treatment until the level of therapeutic immunosuppression can be reduced allowing the recipient's primary T-cell response against CMV to be generated accompanied by an anti-CMV antibody response. ${ }^{1}$ In allogeneic bone marrow transplantation, cytotoxic chemotherapy eradicates 
the leukaemia/lymphoma but also eliminates the recipient's immune system; subsequent infusion of donor bone marrow that contains $\mathrm{T}$ cells requires therapeutic immunosuppression to prevent graft $v$ s host disease GVHD. The risk of CMV retintis is greatest in CMV-positive patients who receive bone marrow from a CMV-negative donor whose $\mathrm{T}$ cells have not encountered CMV before. CMV retinitis occurs at a median 8 months (range 3-12 months) after transplantation, mainly in those $\mathrm{D}-/ \mathrm{R}+$ recipients who have previously had CMV viraemia in the first 3 months after transplantation and who also have chronic GVHD that requires more intense immuno-suppressive treatment.

In HIV infection, CMV retinitis generally occurs in advanced disease when the CD4 $+\mathrm{T}$-cell count is $<50$ cells / $\mu$ l (normal range 500-2000/ $\mu \mathrm{l}$ ), when circulating CMV-specific T cells are still present but their function is greatly reduced. As combination anti-HIV treatment effectively controls HIV replication and restores the number and function of CD4 + T cells (and thereby gives most HIV-infected patients a near normal life expectancy), in countries where effective anti-HIV treatment is available the incidence of HIV-associated CMV retinitis has decreased substantially. After starting anti-HIV treatment, a proportion of patients (typically those whose CD4 + T-cell count is $<50 / \mu \mathrm{l}$ ) develop an immune reconstitution inflammatory syndrome, in which re-awakened $\mathrm{T}$-cell responses against dead or dying organisms cause a paradoxical increase in inflammation and symptoms. Thus, in up to $40 \%$ of HIV-infected patients with CMV retinitis, initiation of anti-HIV treatment leads to immune recovery uveitis, which may include anterior uveitis, cataract, vitritis, cystoid macular oedema, disc oedema, and epiretinal membrane formation; treatment with corticosteroids is often necessary. ${ }^{2}$

\section{Diagnosis of CMV retinitis}

The direct cytopathic effect of CMV produces the characteristic appearance of pale necrotic retina often with focal areas of haemorrhage, in a sectorial distribution spreading centrifugally along vascular arcades; when retinitis is inactive following anti-CMV treatment, the retina remains thin and atrophic associated with clumping of the retinal pigment epithelium. Visual loss is mainly due to direct extension of retinitis to the macula or optic nerve head, or to retinal detachment.

The diagnosis can be confirmed by PCR amplification of viral DNA in specimens of aqueous, with reported sensitivity of close to $100 \%$. In one series of HIVassociated CMV retinitis that compared specimens of aqueous with vitreous, the median concentration of CMV DNA in aqueous (300 copies $/ \mu \mathrm{l}$ ) was 10 -fold lower than in vitreous; the concentration of CMV DNA in aqueous was directly related to the area of active CMV retinitis. $^{3}$

\section{Anti-CMV drug treatment}

After diffusing into CMV-infected cells, Ganciclovir is first phosphorylated by the viral kinase UL97, then further phosphorylated by cellular kinases to Ganciclovir triphosphate, which inhibits the viral DNA polymerase UL54. Valganciclovir is a valine ester of Ganciclovir, which has much better oral bioavailability than Ganciclovir - following intestinal absorption, the valine ester is cleaved to release free Ganciclovir. Cidofovir is phosphorylated by cellular kinases to Cidofovir diphosphate, which inhibits the viral DNA polymerase. As Cidofovir does not require phosphorylation by UL97, Cidofovir remains effective against strains of CMV that have become resistant to Ganciclovir as a result of point mutations in UL97. Cidofovir diphosphate has a very long intracellular half-life that makes possible one infusion every 2 weeks. Foscarnet is a direct inhibitor of the viral DNA polymerase and does not require phosphorylation.

To inhibit the replication of wild-type strains of CMV in vitro, the inhibitory concentration $\mathrm{IC}_{50}$ of Ganciclovir is 0.25-1.22 mg/1. During treatment with intravenous Ganciclovir or oral Valganciclovir, the peak concentration of Ganciclovir in plasma is typically $7.0-9.0 \mathrm{mg} / 1$ whereas the trough plasma concentration is $1.0-2.0 \mathrm{mg} / 1$. The concentration of Ganciclovir in vitreous is typically $0.9-1.2 \mathrm{mg} / \mathrm{l}$, close to the minimum concentration required to inhibit CMV replication. The penetration of Foscarnet from plasma into the vitreous is similarly modest. In contrast, following intra-vitreal injection of Ganciclovir much higher vitreous concentrations are achieved, typically $>20 \mathrm{mg} / 1$ at $72 \mathrm{~h}$ after injection.

During prolonged parenteral therapy of CMV in severely immuno-compromised patients, selection for drug-resistant strains of CMV can occur. Before treatment, $1 \%$ of CMV strains were resistant to Ganciclovir; after 6 months of Ganciclovir treatment, $11 \%$ were resistant; and after 9 months, $27 \%$ were resistant. Before treatment, $2 \%$ of CMV strains were resistant to Foscarnet; after 6 months of Foscarnet treatment, $26 \%$ were resistant; and after 9 months, $37 \%$ were resistant.

\section{Treatment of CMV retinitis}

In large randomized controlled trials of HIV-associated CMV retinitis in the era before combination anti-HIV 
treatment, in which the primary endpoint was objective progression of CMV retinitis, an intra-ocular Ganciclovir implant (15\% of patients progressed after 100 days of treatment) was clearly superior to intravenous Ganciclovir (65\% of patients progressed). ${ }^{4}$ The limitation of the intra-ocular Ganciclovir implant was its failure to prevent CMV disease in the contralateral eye. In a subsequent randomized controlled trial of HIV-associated CMV retinitis, treatment with oral Valgaciclovir (38\% of patients progressed after 100 days of treatment) was similarly effective to initial intravenous Ganciclovir for 4 weeks followed by oral Valganciclovir (45\% of patients progressed). ${ }^{5}$ During the latter trial, most patients were also taking combination anti-HIV treatment. As the ocular penetration of systemically administered anti-CMV drugs is limited, current clinical guidelines include consideration of intra-ocular injection of anti-CMV drugs for patients who have sight-threatening CMV retinitis.

\section{CMV anterior segment disease}

In the last 5 years, the involvement of CMV has been increasingly recognized in two forms of anterior segment disease, in both of which CMV DNA is detected in aqueous by PCR amplification. In the pathogenesis, it remains unclear whether CMV reactivation is the primary cause of the inflammation or whether local CMV reactivation occurs in the anterior segment as a secondary consequence of macrophage/ dendritic cell activation provoked by a different primary cause (as for example, local CMV reactivation occurs in the large intestine in some patients who have ulcerative colitis or Clostridium difficile-associated colitis).

\section{CMV anterior uveitis}

The clinical presentation is unilateral recurrent acute or chronic anterior uveitis usually associated with raised intra-ocular pressure in an immuno-competent adult. ${ }^{6}$ The anterior uveitis may not respond to topical steroids. There is generally a clinical response to systemic or intra-vitreal anti-CMV treatment, but inflammation frequently recurs after the anti-CMV treatment is stopped; the most effective treatment strategy for this recurrent inflammation is unclear, and a clinical trial is needed.

\section{CMV corneal endotheliitis}

The clinical presentation is corneal endothelial cell loss, local stromal oedema of the cornea and keratic precipitates, sometimes accompanied by raised intraocular pressure or iris atrophy in an immuno-competent adult. $^{7}$ A higher CMV viral load in aqueous has been associated with increased corneal endothelial cell loss. In some reports, the development of CMV endotheliitis was preceded by local or systemic immunosuppression or penetrating corneal surgery. In most cases, systemic anti-CMV treatment has been followed by improvement in endotheliitis and improvement in intra-ocular pressure. As corneal endotheliitis can also be related to HSV or VZV reactivation, an accurate virological diagnosis by PCR amplification of aqueous is necessary to ensure the correct antiviral treatment.

\section{Conflict of interest}

The author declares no conflict of interest.

\section{References}

1 Eid AJ, Bakri SJ, Kijpittayarit S, Razonable RR. Clinical features and outcomes of cytomegalovirus retinitis after transplantation. Transplant Infect Dis 2008; 10: 13-18.

2 Karavellas MP, Azen SP, MacDonald JC, Shufelt CL, Lowder $\mathrm{CY}$, Plummer DJ et al. Immune recovery vitritis and uveitis in AIDS; clinical predictors, sequelae and treatment outcomes. Retina 2001; 21: 1-9.

3 Smith IL, Macdonald JC, Freeman WR, Shapiro AM, Spector SA. Cytomegalovirus (CMV) retinitis activity is accurately reflected by the presence and level of CMV DNA in aqueous humor and vitreous. J Infect Dis 1999; 179: $1249-1253$.

4 Musch DC, Martin DF, Gordon JF, Davis MD, Kuppermann BD, Ganciclovir Implant Study Group. Treatment of cytomegalovirus retinitis with a sustained-release ganciclovir implant. N Engl J Med 1997; 337: 83-90.

5 Martin DF, Sierra-Madero J, Walmsley S, Wolitz RA, Macey K, Georgiou P. A controlled trial of valganciclovir as induction therapy for cytomegalovirus retinitis. N Engl J Med 2002; 346: 1119-1126.

6 van Boxtel LA, van der Lelij A, van der Meer J, Los LI. Cytomegalovirus as a cause of anterior uveitis in immunocompetent patients. Ophthalmology 2007; 114: 1358-1362.

7 Koizumi N, Suzuki T, Uno T, Chihara H, Shiraishi A, Hara Y et al. CMV as an etiologic factor in corneal endotheliitis. Ophthalmology 2008; 115: 292-297. 